\title{
Análisis Facial, Dentario y Radiográfico de la Normalidad Facial. Estudio Piloto en 29 Mujeres
}

\author{
Facial, Dental and Radiographic Analyses of Facial Normality. A Pilot Study in 29 Women
}

\author{
Claudio Huentequeo-Molina*; Pablo Navarro**; Bélgica Vásquez**** \& Sergio Olate*
}

HUENTEQUEO-MOLINA, C.; NAVARRO, P.; VÁSQUEZ, B. \& OLATE, S. Análisis facial, dentario y radiográfico de la normalidad facial. Estudio piloto en 29 mujeres. Int. J. Morphol., 31(1):150-155, 2013.

RESUMEN: La estética y armonía facial se relacionan de forma directa con la percepción y autoestima de los individuos; muchas veces se generan deseos de cambios estéticos por medio de cirugías para obtener una valoración positiva de sí mismo. El objetivo de este estudio fue investigar la relación entre la percepción, autoestima y deseo de cambio estético; y puntos antropométricos, cefalométricos y maloclusiones de un grupo de mujeres. Se escogieron 29 mujeres chilenas, entre 20 y 25 años de edad, en las cuales se realizó una encuesta sobre la autoestima, autopercepción de normalidad facial, deseo de cambio estético y cambio de autoestima posterior a una cirugía, éstas se asociaron a través de un análisis de chi2, regresión logística de multivariado y ANOVA, con las distancias entre puntoantropométricos objetivos basados en estudios de Farkas y entre puntos cefalométricos basados en Epker y Fish; y entre ellas a través de un análisis. La normalidad facial se ve afectada principalmente por clases caninas derecha $(\mathrm{p}=0,02)$ e izquierda $(\mathrm{p}=0,015)$ y molares derecha $(\mathrm{p}=0,015)$ e izquierda $(\mathrm{p}=0,04)$; y además el apiñamiento dentario $(\mathrm{p}=0,012)$. Mientras el aumento en la distancia de exocantios $(\mathrm{p}=0,04)$, bases alares $(\mathrm{p}=0,03)$, proporción glabela subnasal y subnasal mentón $(\mathrm{p}=0,02)$ se asocian a una percepción de anormalidad. La autoestima puede variar de forma positiva posterior a un cambio estético, a través de cirugía o tratamiento de ortodoncia. Los parámetros dentomaxilares y craneofaciales son determinantes en la percepción de normalidad estética de estas mujeres, siendo los relacionados con nariz y altura facial los de mayor influencia en este estudio. Son necesarios futuros estudios para evaluar autoestima, autopercepción de normalidad facial y proporciones estéticas.

PALABRA CLAVE: Estética facial; Antropometría facial; Cefalometría

\section{INTRODUCCIÓN}

En el área maxilofacial, la antropometría y posteriormente la cefalometría han sido utilizadas por diversos autores a fin de determinar la normalidad y la estética facial (Farkas 1981, Epker \& Koury, 1992). Este análisis es fundamental en la cirugía oro-facial moderna ya que hoy día son demandadas e indicadas intervenciones quirúrgicas donde la auto-percepción estética es más determinante que la auto-percepción de una maloclusión o incluso que el diagnóstico clínico de una anormalidad facial (Phillipsa \& Beal, 2009).

Determinar estética y armonía facial es complejo y controversial; se propuso inicialmente la "proporción divina" como un elemento de análisis, mientras que otros sugirieron disponer de promedios o cánones faciales que sean considerados estéticos (Farkas et al., 1985 y Galton 1978). Así, si bien existen patrones de normalidad (Farkas \& Kolar,
1987), es posible observar sujetos autodefinidos como normales y cuyos patrones faciales están fuera de la normalidad o también sujetos en quienes el rostro posee cualitativamente algunas características anormales y que por sus pares no son considerados anormales (Rubenstein et al. 2001, Komori et al. 2009)

Las disciplinas que basan su estructura de trabajo clínico en modificaciones faciales debido a trauma, patología o modificaciones cosméticas deben incorporar elementos de la estética facial en su diagnostico (Farkas \& Munro, 1987 y Epker et al., 1993), a fin de obtener planificaciones integrales en los tratamientos propuestos. El objetivo de esta investigación fue determinar la relación entre la auto-percepción de la normalidad facial, los patrones faciales y los patrones dentoesqueletales de mujeres de entre 20 y 25 años autodefinidas como normales.

\footnotetext{
Programa de Magíster en Odontología, Facultad de Odontología, Universidad de La Frontera. Unidad de Cirugía Oral y Maxilofacial, Facultad de Odontología, Universidad de La Frontera, Temuco, Chile.

** Departamento de Matemáticas y Estadística, Facultad de Ingeniería Ciencias y Administración, Universidad de La Frontera, Temuco, Chile.

**** Universidad de Tarapacá, Arica, Chile.
} 


\section{MATERIAL Y MÉTODO}

Se realizó un estudio descriptivo transversal e inferencial, donde se escogió a 29 sujetos chilenos del sexo femenino de entre 20 y 25 años de edad que accedieron de forma voluntaria al estudio; fueron excluidas mujeres embarazadas, con enfermedades siquiátricas, con antecedentes de trauma facial, presencia actual o pasada de tratamiento de ortodoncia, presencia de cirugía cosmética, reparadora o reconstructiva facial y pérdida o agenesia dentaria (no incluidos los terceros molares). Todos los sujetos firmaron un documento de consentimiento informado donde se explicaban los alcances de la investigación.
Los sujetos incluidos en la investigación debían ser auto percibidos como normales al momento de responder un cuestionario que se elaboro a partir de preguntas presentes en la literatura internacional y a la sugerencia de un comité de expertos donde la respuesta a cada pregunta tenía una valoración del 1 al 5 (Tabla I). El objetivo de esta encuesta fue determinar la autopercepción de normalidad del sujeto seleccionado. La encuesta se realizó en un box odontológico con adecuada iluminación.

En una segunda oportunidad, en el mismo lugar, se realizó el estudio de análisis facial (Figs. 1 y 2); el sujeto de estudio se posicionó sentado en angulación de $90^{\circ}$ y con la visión hacia anterior, manteniendo el plano bipupilar parale-

Tabla. I. Preguntas utilizadas para identificar los niveles de percepción del sujeto de muestra en las 29 mujeres estudiadas con edades entre 20 a 25 años.

\begin{tabular}{|c|c|c|c|c|c|c|}
\hline ITEM & Pregunta & 1 & 2 & 3 & 4 & 5 \\
\hline Autoestima & ¿Cómo consid era su autoestima? & & & & & \\
\hline Estética & ¿Ud. se encuentra dentro de la normalidad estética facial? & & & & & \\
\hline Cambio estético & ¿Cambi aría algo en su cara, a tra vés de una cirugía, tratamiento de ortodoncia u otro? & & & & & \\
\hline Cambio Autoestima & ¿Cree Ud. que este cambio favorecería su autoestima? & & & & & \\
\hline
\end{tabular}

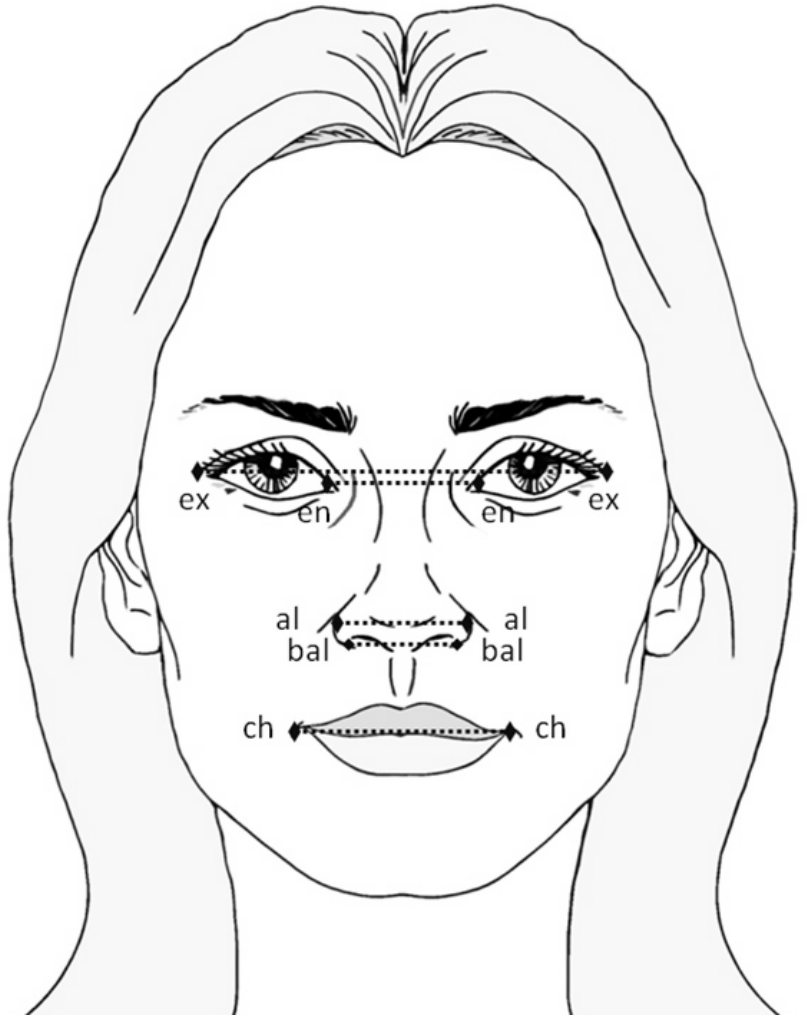

Fig. 1 Ubicación espacial de los puntos antropométricos utilizados en el análisis facial de los 29 individuos de sexo femenino con edades entre 20 a 25 años.

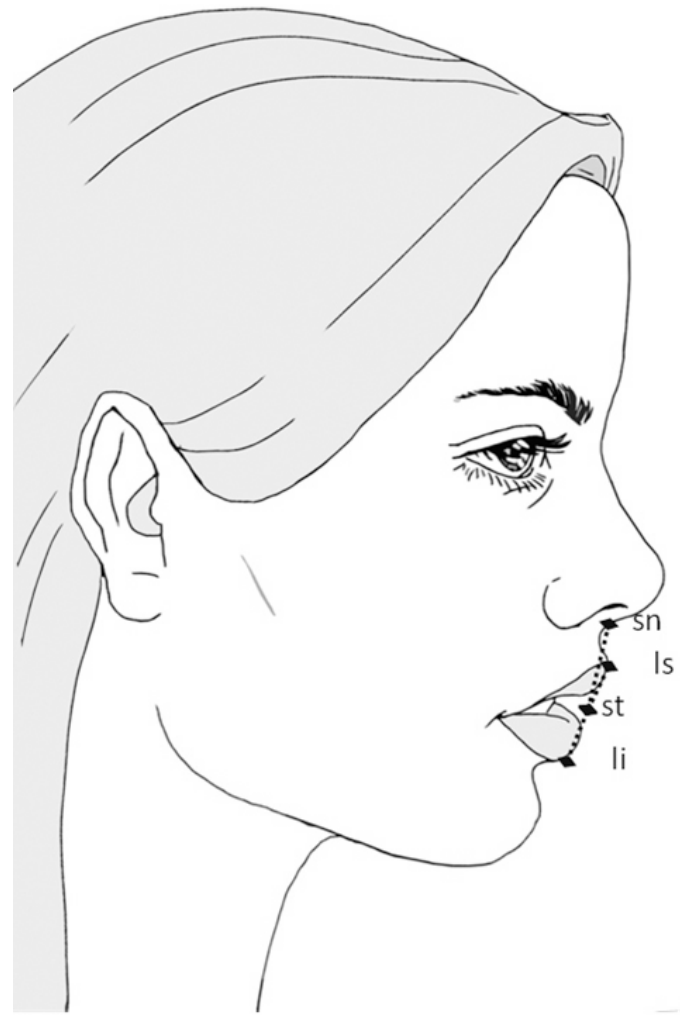

Fig. 2. Ubicación espacial de los puntos utilizados a nivel del punto subnasal, labio superior e inferior en análisis facial lateral. 
lo al piso; para las mediciones se utilizó una regla flexible milimetrada en los puntos seleccionados. Previamente, existió una calibración intra e interoperador con medición de 10 sujetos previo al estudio a fin de calibrar los dos observadores que realizarían esta evaluación. Los puntos analizados se resumen en la Tabla II y se originan de las definiciones propuestas por Epker (1993).

En la misma etapa se realizó el análisis dentario donde se estudió la clase canina y molar bilateral, existencia del apiñamiento dentario, diastema y línea de sonrisa. Posteriormente se realizó una radiografía lateral (equipo Modelo: PantOs 16 Ceph. Panoramic \& Cephalometric Dental X-Ray Sistem. Marca Bluex Año 2004, Assago-Italy), la posición del sujeto también presentaba el plano bipupilar paralelo al piso, el que fue orientado por el mismo observador que realizaba el análisis facial previo (Figs. 3 y 4). El estudio de la imagen radiográfica incluyó los puntos resumidos en la Tabla III según lo propuesto por Epker (1993).

Análisis estadístico. Inicialmente, se realizó un análisis descriptivo de cada variable. Posteriormente, se realizó la prueba de normalidad de estas medidas a través de la prueba de

Tabla II. Puntos y distancias utilizadas para obtener las medidas faciales en tejidos blandos de 29 individuos de sexo femenino de entre 20 a 25 años.

\begin{tabular}{lcc}
\hline Distancias en punto de análisis & Abreviatura & Valores normales (Epker \& Koury 1992) \\
\hline Subnasal- Estomion & $\mathrm{Sn}-\mathrm{St}$ & $20.1(+-2.0)$ \\
Labio inferior - Stomion & $\mathrm{St}-\mathrm{Li}$ & $9.4(+-1.5)$ \\
Labio superior - Estomion & $\mathrm{Ls}-\mathrm{St}$ & $8.7(+-1.3)$ \\
Exocantium - Exocantium & $\mathrm{Ex}-\mathrm{Ex}$ & $86.8(+-2)$ \\
Endocantium - Endocan tium & $\mathrm{En}-\mathrm{En}$ & $31.8(+-2.3)$ \\
Alar - Alar & $\mathrm{Al}-\mathrm{Al}$ & $31.4(+-2.0)$ \\
Base alar - Base alar & $\mathrm{bAl}-\mathrm{bAl}$ & $30.5(+-2.2)$ \\
Cheilon- Cheilon & $\mathrm{Ch}-\mathrm{Ch}$ & $50.2(+-3.5)$ \\
\hline
\end{tabular}

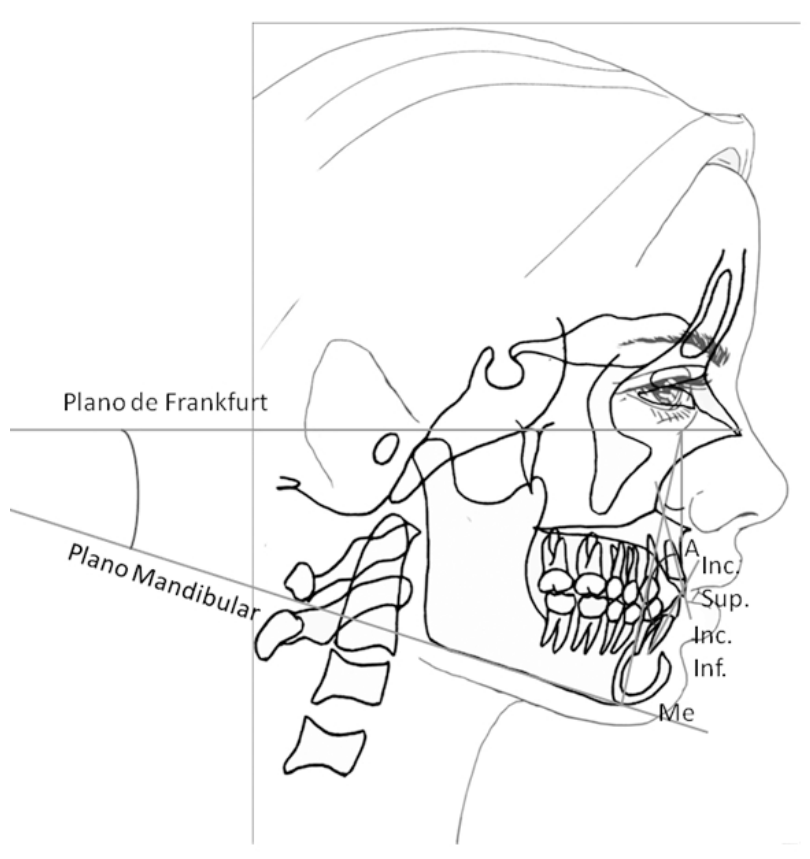

Fig. 3. Planos y ángulos utilizados en tejido óseo para en el análisis de radiografía lateral de los 29 individuos de sexo femenino de entre 20 a 25 años.

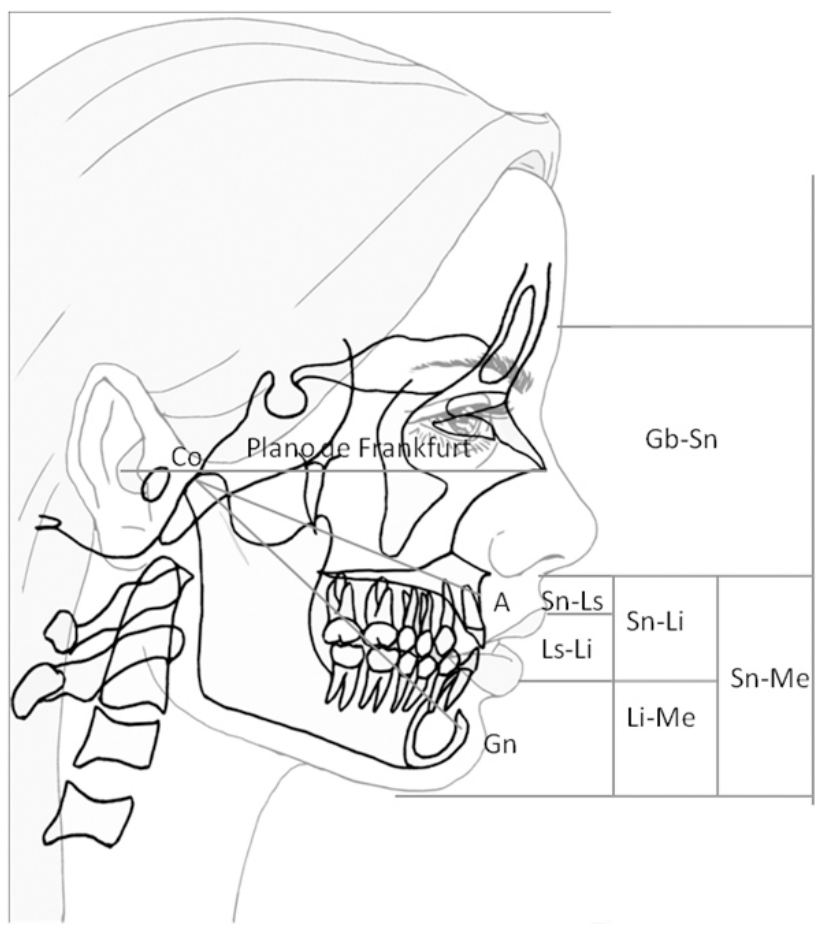

Fig. 4. Planos, distancias y ángulos utilizados en tejido blando para el análisis de la radiografía lateral de 29 sujetos de sexo femenino de entre 20 a 25 años 
Shapiro- Wilk. Se evalúo el nivel de influencia de las variables cualitativas mediante el análisis factorial de correspondencias múltiples y el análisis de segmentación jerárquica. Se realizó el análisis de distribución Chi-Cuadrado con el fin evaluar la asociación entre la auto-percepción de normalidad estética y los patrones dentomaxilares. Las variables relacionadas con las medidas se asociaron a través de un análisis de regresión logística binaria. Cuando estas variables se relacionaron con el nivel de autoestima, se realizó un análisis ANOVA para medir la asociación de éstas. El análisis estadístico en esta investigación fue realizado a través del programa SPSS Inc., Chicago, IL, USA.

Tabla III. Distancias y ángulos utilizados para obtener las medidas faciales en tejidos óseos y blandos en radiografías laterales de 29 individuos de sexo femenino de entre 20 a 25 años.

\begin{tabular}{|c|c|c|}
\hline Puntos Antropométricos Lineares y Angulares & Abreviatura & Valores normales \\
\hline Glabela - Subnasal & G-Sn & $70.7(+-3.5)$ \\
\hline Subnasal - Labio inferior & Sn-Li & --- \\
\hline Condilion - Punto A & Co-pA & --- \\
\hline Perpendicular PF subnasal - Labio superior & Per. Sn tej. B1.- Ls & $0(+-2)$ \\
\hline Perpendicular PF subnasal - Labio inferior & Per. Sn - Li & $-2(+-2)$ \\
\hline Perpendicular PF subnasal - Mentón & Per. Sn - Me & $-4(+-2)$ \\
\hline Subnasal - Mentón & $\mathrm{Sn}-\mathrm{Me}$ & $69.7(+-3.3)$ \\
\hline Labio Inferior - Mentón & $\mathrm{Li}-\mathrm{Me}$ & --- \\
\hline Condilion - Gnation & Co- $\mathrm{Gn}$ & --- \\
\hline $\mathrm{PF}-\mathrm{Me} / \mathrm{PF}$ - Punto A & & $89+3^{\circ}$ \\
\hline PF-Me / Plano Mandibular & & $90+-3^{\circ}$ \\
\hline PA incisivo superior / PA incisivo inferior & & $130+-6^{\circ}$ \\
\hline
\end{tabular}

\section{RESULTADOS}

Los sujetos de investigación fueron evaluadas sin alteraciones al protocolo de trabajo propuesto. En las evaluaciones de patrones dentales la presencia de diastemas no tuvo asociación significativa con la percepción de estética facial ( $\mathrm{p}=0,077)$. Quienes presentaban patrones alterados de clase canina derecha $(p=0,02)$ e izquierda $(\mathrm{p}=0,015)$ y molares derecha $(\mathrm{p}=0,015)$ e izquierda $(p=0,04)$ e incluso el apiñamiento dentario $(p=0,012)$ fueron asociados a una mayor sensación de estética facial anormal. El tipo de sonrisa no fue asociada a parámetros de anormalidad facial.

Las mediciones antropométricas demostraron que al aumentar la distancia entre exocantios hay mayor asociación con anormalidad facial ( $\mathrm{p}=0,04)$; de igual forma, al aumento de la distancia entre los puntos de base alar derecha e izquierda también se asocia a la anormalidad facial $(\mathrm{p}=0,03)$. La distancia entre el punto subnasal y el estomion no se vincularon a la percepción de anormali$\operatorname{dad}(\mathrm{p}=0,06)$.

En las mediciones realizadas en radiografías late- rales se demostró que leves variaciones en la proporción glabela - subnasal / subnasal - mentón generan una fuerte asociación con anormalidad facial $(\mathrm{p}=0,02)$; la distancia entre el punto subnasal y el punto mentón, por si sola, no está asociada a alteraciones de la normalidad facial ( $\mathrm{p}=0,996)$ y el análisis del ángulo compuesto por los puntos condilion - punto A y los puntos condilion - gnation determinaron un bajo riesgo de percepción de anormalidad.

Las variables autoestima, cambio de autoestima, normalidad facial y deseo de cambio estético fueron evaluadas a través de un análisis de segmentación jerárquico donde se determinó la relación de estas variables y sus influencias. La autoestima fue influenciada principalmente por el deseo de cambio estético; de hecho, el 53,7\% de los sujetos declararon que su autoestima tendría un cambio positivo si fuesen sometidos a algún cambio estético facial. Sin embargo, las medidas analizadas individualmente tanto en el área dental, facial o radiográfica no presentaron relación significativa con el autoestima del sujeto. 


\section{DISCUSIÓN}

La selección de mujeres en esta investigación se asocia al hecho de que frecuentemente se perciben de forma más positiva en cuanto a su belleza o normalidad al compararlas con lo que otros observadores pueden describirlas (Cazzato et al., 2012). Los estándares de normalidad facial son distintos para cada etnia, cultura, región del mundo o condición socioeconómica (Leong \& White, 2006; Husein et al., 2010 y Cazzato et al.) y aun así son utilizados prácticamente los mismos estudios de análisis facial y estudios cefalómetros en diferentes puntos del mundo y en diferentes grupos de poblaciones.

La armonía facial ha sido estudiada a través de la historia y muchos autores la proponen como pilar fundamental de la belleza (Jahanbin et al. 2008). Siendo un concepto subjetivo, se puede hacer algo más objetiva gracias a las mediciones antropométricas (Farkas 1981, 1985) y cefalométricas (Epker), aunque la armonía y los promedios de estas medidas dependen de cada población analizada (Galton; Rhodes 2006). De hecho, el prototipo facial común para cada población, tiene directa relación con la belleza percibida por los demás individuos (Rubenstein et al.,) y quienes se acercan a ese promedio son percibidos como más atractivos (Komori et al.).

Una de las desventajas de las medidas faciales es que muchas veces se hacen sobre puntos antropométricos de difícil localización, generando sesgos en las mediciones (Epker \& Koury 1992, Altug-Ataca et al., 2008). En este estudio, se intento disminuir el error utilizando puntos de fácil ubicación, determinados por la presencia de otros elementos anatómicos de referencia; de esta forma se incluyó puntos como el exocantion y se excluyeron puntos como el zygion.

Por otra parte, la exageración de las anomalías y asimetrías faciales desencadenan de forma inminente la percepción de anormalidad facial, de forma que en este estudio sólo se relacionó medidas que siendo anormales, solo fueran de forma leve (1-3 mm).

Los sujetos de estudio, según el cuestionario aplicado, se consideraron normal en términos de patrones faciales. La autoestima en ellas, sin embargo, fue definida como muy buena solo en un $17 \%$, buena en un $48 \%$ y regular en un $35 \%$, demostrando la variabilidad de condicionantes que pueden observarse en pacientes autodefinidas como facialmente normales. En este grupo, la autoestima es principalmente influida por el deseo de un cambio estético, ya sea a través de una cirugía facial o tratamiento de ortodoncia (ningún sujeto tenía tratamiento quirúrgico o de ortodoncia previo). A pesar de esto, encontramos ángulos, proporciones cefalométricas y antropométricas que no influyen directamente en la estética facial y si otras medidas que logran mayor relevancia en la belleza o estética facial (Kiekens et al., 2008). Dentro de estos cambios estéticos, uno de los más valorados son las variaciones morfológicas de la nariz, que de acuerdo a otros autores (Husein et al.), genera una alta demanda de rinoplastías; en este sentido, la anatomía nasal muchas veces es condicionada por la posición de la maxila (espina nasal anterior), más que por la forma de los huesos propios nasales o el septo nasal (también asociadas a las clases esqueletal y dentaria). La percepción de normalidad de las mujeres de este estudio se ve afectada por las maloclusiones, pero coincidiendo con lo que plantea Kiekens et al., existen algunos patrones que no generan gran impacto en el deseo de cambio estético; de esta forma, sujetos con alteración en las clases dentarias o presencia de apiñamiento dentario, tienen más riesgo de sentirse facialmente anormales.

Las distancias más significativas en cuanto a la apreciación de su propia normalidad estética (de las utilizadas en esta investigación) se relacionan con el ancho de la nariz (alar-alar y base alar-base alar), concordando con lo reportado por Husein et al., y la distancia de los exocantios y endocantios y las proporciones verticales faciales (Epker \& Koury, 1992). Finalmente podemos concluir que la autoestima y armonía facial son fundamentales para determinar necesidad de cirugía; medidas aisladas no necesariamente van a influenciar en la condición de anormalidad dento-facial. Estudios con mayor población y con otro tipo de muestras son necesarias para determinar las condicionantes en población chilena.

HUENTEQUEO-MOLINA, C.; NAVARRO, P.; VÁSQUEZ, B. \& OLATE, S. Facial, dental and radiographic analyses of facial normality. A pilot study in 29 women. Int. J. Morphol., 31(1):150$155,2013$.

SUMMARY: Aesthetics and facial harmony are directly related to self-esteem and perception individuals have of themselves. There is often a desire to make overall aesthetic changes through surgery in order to get a positive assessment of oneself. The aim of this study was to research the relationship between perception, self-esteem and desire for change; and points aesthetic anthropometric and cephalometric points, as well as malocclusions in a group of women. Twenty nine (29) Chilean women between 20 and 25 years of age were chosen, taking part in a survey on self-esteem, self-perception of facial normalcy, the desire for aesthetic change, and changes of self-esteem following surgery. These were subsequently associated through $\mathrm{Chi}^{2}$ analysis, 
logistic multi variant regression and ANOVA, with distances between objective anthropometric marks based on Farkas research, and between cephalometric points based in Epker and Fish study. Facial normalcy seems mainly affected by right canine class $(\mathrm{p}=0.02)$, and left $(\mathrm{p}=0.015)$; right molar class $(\mathrm{p}=0.015)$ and left $(\mathrm{p}=0.04)$, as well as dental crowding $(\mathrm{p}=0.012)$. While greater exocanthion distance $(\mathrm{p}=0.04)$, alar base $(\mathrm{p}=0.03)$, subnasal glabella and subnasal menton ratios $(\mathrm{p}=0.02)$, are related to a perception of abnormality. Self esteem perception can vary in positive ways following an aesthetic change through surgery or orthodontic treatment. Dental, maxillary and craniofacial parameters are determinants of the aesthetic normalcy perception of these women, with nose and facial height having the most influence in this study. Further research is necessary to evaluate self- esteem and self perception of facial normalcy, as well as aesthetic ratios.

\section{Cephalometry.}

KEY WORD: Facial aesthetics; Facial anthropometry;

\section{REFERENCIAS BIBLIOGRÁFICAS}

Altug-Ataca, A.; Bolatoglub, H. \& Memikogluc U. Facial Soft Tissue Profile Following Bimaxillary Orthognathic Surgery. Angle Orthod., 78(1):50-7, 2008.

Cazzato, V.; Siega, S. \& Urgesi C. What women like: influence of motion and form on esthetic body perception. Front Psychol., 3:235, 2012.

Epker, B.N. \& Koury, M. Maxillofacial Esthetics: Anthropometrics of the Maxillofacial Region. J. Oral Maxillofac. Surg., 50(8):606-20,1992.

Epker, B.N.; Fish, L. \& Sullivan, C. Orthognathic Surgery: The Correction of Dentofacial Deformities. J. Oral Maxillofac. Surg., 51 (1 SuppI 1):28-41, 1993.

Farkas, L.G. Anthropometrics of the Head and Face in Medicine. New York, NY, Elsevier, pp 8-59, 108-202, 1981.

Farkas, L.G.; Hreczko, T. A. \& Kolar, J.C. Vertical and horizontal proportions of the face in young adult North American caucasians: Revision of neoclassical canons. Plast. Reconstr. Surg., 75:(3)328-38, 1985[??2] .

Farkas, L.G. \& Kolar, J.C. Anthropometrics and art in the aesthetics of women's faces. Clin. Plast. Surg., 14(4):599-616, 1987.

Farkas, L.G. \& Munro, I.R. Anthropometric Facial Reconstruction in Medicine. Springfield, IL, Thomas, pp 3-8, 19-26, 49-55, 119-129, 166-319, 1987.

Galton, F. Composite portraits, made by combining those of many different persons into a single resultantfigure. J. Anthropol Inst Great Brit Ireland, 8:132-44, 1879.
Husein, O.; Sepehr, A.; Garg, R.; Sina-Khadiv, M.; Gattu, S.; Waltzman, J.; Wu, E.; Shieh, M.; Heitmann, G. \& Galle, S. Anthropometric and aesthetic analysis of the Indian American woman's face. J.P.R.A.S., 63(11):1825-31, 2010.

Jahanbin, A.; Basafa, M. \& Alizadeh, Y. Evaluation of the Divine Proportion in the facial profile of young females. Indian. $J$. Dent. Res., 19(4):292-6, 2008.

Kiekens, R.; Kuijpers-Jagtman, A.; Van’t Hof, M.; Van’t Hof B.; Straatman H. \& Maltha J. Facial esthetics in adolescents and its relationship to ideal ratios and angles. Am. J. Orthod. Dentofacial. Orthop.,133(2):188.e1-188.e8, 2008.

Kiekens, R.; Kuijpers-Jagtman, A.; Van't Hof, M.; Van’t Hof B.; Maltha, J. Putative golden proportions as predictors of facial esthetics in adolescents. Am. J. Orthod. Dentofacial. Orthop., 134(4):480-3, 2008.

Komori, M.; Kawamura, S. \& Ishihara, S. Averageness or symmetry: Which is more important for facial attractiveness? Acta Psychologica, 131(2):136-42, 2009.

Leong, S. \& White, P. A comparison of aesthetic proportions between the healthy Caucasian nose and the aesthetic. J.P.R.A.S., 59(3):248-52, 2006.

Phillipsa, C. \& Beal K.N. Self-Concept and the Perception of Facial Appearance in Children and Adolescents Seeking Orthodontic Treatment. Angle Orthod.,79(1):12-6, 2009.

Rhodes, G. The evolutionary psychology of facial beauty. Annual Review of Psychology, 57:199-226, 2006.

Rubenstein, A.J.; Langlois, J.H. \& Roggman, L. What makes a face attractive and why: The role of averageness in defining facial beauty. In: Rhodes, G.; Zebrowitz, LA., editors. Advances in visual cognition. Westport, CT: Ablex; vol. 1, p. $1-33,2001$.

\section{Correspondencia}

Prof. Dr. Sergio Olate

Unidad de Cirugía Oral y Máxilofacial

Facultad de Ondotología

Universidad de La Frontera

Claro Solar 115

CHILE

Teléfono: 56-45-325000

Email: sergio.olate@ufrontera.cl

Recibido : 18-11-2012

Aceptado: 22-12-2012 